\title{
A NEW REGION-BASED ADAPTIVE THRESHOLDING FOR SPERM MOTILITY SEGMENTATION
}

\author{
Khairunnisa Hasikin ${ }^{1}$, Nor Ashidi Mat Isa ${ }^{2}$, Mahaneem Mohamed ${ }^{3}$, \\ Shahrel Azmin Suandi', Nahrizul Adib Kadri ${ }^{5}$ \\ ${ }^{1,5}$ Department of Biomedical Engineering, Faculty of Engineering, \\ University of Malaya, 50603, Lembah Pantai, Kuala Lumpur, Malaysia \\ 1, 2, 4 Imaging and Intelligent Research Team (ISRT), School of Electrical and Electronic Engineering, \\ Engineering Campus, Universiti Sains Malaysia, 14300, Nibong Tebal, Penang, Malaysia. \\ ${ }^{3}$ Department of Physiology, School of Medical Sciences, Health Campus, \\ Universiti Sains Malaysia, 16150, Kubang Kerian, Kelantan, Malaysia. \\ Email: khairunnisa@um.edu.my¹,ashidi@usm.my, shahrel@usm.my³,mahaneem@usm.my², \\ nahrizuladib@um.edu.my ${ }^{5}$
}

\begin{abstract}
Infertility cases have shown increasing growth in recent years where approximately $40 \%$ of root causes of infertility cases are related to men. Researchers have shown that the sperm motility has significantly contributed towards infertility as compared to its concentration and morphology. However, existing technique has faced difficulties in segmenting motile sperm in the low contrast regions. The movements of the motile sperms that are normally fast further complicate the automated segmentation. In this paper, we present a new region-based adaptive thresholding technique that consists of four main stages. Pixels of the images are classified based on the intensity distribution and those pixels are grouped and processed separately. Multiple thresholds are generated based on the classified group to ensure objects in low contrast regions are segmented. In addition, the proposed method does not require external pre-processing tool or phase contrast accessories prior to the sperm segmentation. Our experimental evaluations show that the proposed method produces significant improvement from the existing technique with the average accuracy of $95.74 \%$. The qualitative results also indicate that the proposed method is able to segment the motile sperms in the low contrast region. These results of sperm segmentation are in agreement with the quantitative measurement of non-uniformity where the proposed method attains lower non-uniformity with respect to the results achieved by the other method.
\end{abstract}

Keywords: sperm motility, segmentation, thresholding, sperm detection, semen analysis.

\subsection{INTRODUCTION}

Male factor infertility cases caused by defective sperm parameter had increased in recent years. Infertility evaluation plays a major role in identifying the underlying cause of this issue. Semen analysis is an intial and most essential step of male factor infertility evaluation. It provides prognostic information for fertility potential. The evaluation included a physical examination, hormonal evaluation, sperm parameter testing and genetic analysis. The seminal concentration, sperm motility and morphology have been used as biomarkers of male infertility since the middle of the 20th century [1]. 
However several studies have concluded that the sperm motility is highly contributed to the fertilization rate either in vivo or in vitro than relying only on the sperm count or morphology of the sperm [2-4]. The identification of sperm motility has potential importance in sperm function tests and in reproductive toxicology investigation. Sperm motility is a critical indicator of semen quality and fertility potential since it is required for the penetration of cervical mucus. It becomes critical during fertilization since it allows and facilitates the passage of the sperm through the egg membrane. The advancement in imaging technology has enabled the analysis of sperm behavior through microscopic imaging. In this approach, images which have been acquired from semen specimens are analyzed manually by an expert person. An automated method has been utilized as substitute of manual analysis particularly to measure important parameters of sperms. Tracking a large number of sperms by eye is difficult and time-consuming procedure where visual problems and fatigue can affect negatively on the result. A good estimation on sperm tracking can be obtained by utilizing an effective automated segmentation tool to segment the region of interest (i.e. motile sperm). The dynamic movement of the sperm in consecutive frames has increased the complexity of sperm tracking due to the changes in the sperm movement orientation. In addition, poor quality of images that suffer from non-uniform illumination and low contrast further complicates the automated segmentation and detection of the motile sperm. Therefore, with the existing limitations, many hospitals in Malaysia and developed countries are still relying on manual approach.

In this paper, a new method of sperm segmentation is introduced which is based on the thresholding technique. The remainder of this paper is organized as follows. Section 2 describes the related works that proposing segmentation technique in sperm characteristic analysis. In Section 3, the proposed methodology is presented where details of the multi stages segmentation are given. Sections 4 and 5 describe the results and discussions respectively. Finally in Section 5, conclusions are drawn and future work is outlined.

\subsection{RELATED WORKS}

Sperm detection analysis is relying on how discriminative features of the sperms are segmented for sperm identification. These segmented features of the sperm (i.e. either whole body of the sperms or sperm's head) play a major role in sperm morphology and motility analysis. Computer Assisted Sperm Analysis (CASA) systems offer morphological analysis and few provide tracking analysis of the sperm such as Hamilton-Thorne Sperm Analyzer (Beverly, MA, USA)[5], Sperm Class Analyzer (SCA - Microoptics, Barcelona Spain)[6] and medeaLAB (Medical Technology MTG, Altdorf Germany)[7]. These systems have difficulties in rendering sperm tails where some cases the agglutinated or clumped sperms are unidentified and removed from analysis. The high cost, specialized equipment and expert technician required to operate them make these systems difficult to be introduced into andrological practice. In addition, some of the available CASA systems require additional phase-contrast accessories to aid the identification of the sperm. The aforementioned disadvantages have increased the complexity of the data interpretation and remark the need of objective, precise and repeatable techniques to study movement of the sperm. Therefore, the development of new methodologies is actively conducted where automated or semi-automated approaches have been proposed by researchers to track and identify human sperms [7-15], rat sperms [16, 17], and boar sperms [18].

The identification of the sperms is mainly depended the segmentation process to distinguish the sperms from their background. Some researchers focused on segmentation and detection of the sperm's acrosome that located on its head while others relying on the complete structure of the sperm (i.e. head, mid-piece and tail) as listed in Table 1. This table listed segmentation method used to segment the sperm from its background. There are few segmentation approaches required pre-processing to improve its quality before the object of interest was segmented. Smoothing filter such as Gaussian and mean filter were utilized to remove the unwanted noises [7, 11, 15, 16, 19, 20]. The work of Ravanfar, et al. [12] utilizes morphological filtering to improve quality of the image by enhancing the discriminative features. Researchers such as Carrillo, et al. [10] and Vahid Reza, et al. [15] also employ enhancement techniques prior to the segmentation process. 
A two-dimensional maximum entropy method was presented by Guihai, et al. [11] to calculate global threshold for sperm segmentation. The edges of the sperm were extracted from Canny edge detector. The features in this technique determined the threshold of the image and thus ensure its high accuracy. Ravanfar, et al. [12] utilized watershed segmentation algorithm based on topography of intensity. Each area in the image was divided into three categories namely minimum regions, catchman basin and watershed lines. The authors also employed Otsu thresholding at the local region around the single target to avoid over-segmentation. Other authors such as Abbiramy and Shanthi [21], Bijar, et al. [9] and Carrillo, et al. [10] also proposed segmentation algorithms but their focuses are more on sperm morphological analysis.

Table 1: Summary of relevant research works for sperm segmentation and identification

\begin{tabular}{|c|c|c|c|c|c|}
\hline \multirow[t]{2}{*}{ Authors } & \multirow[t]{2}{*}{ Methods } & \multicolumn{3}{|c|}{$\begin{array}{l}\text { Detected Features of the } \\
\text { sperm }\end{array}$} & \multirow[t]{2}{*}{ Pre-processing } \\
\hline & & Head & $\begin{array}{l}\text { Mid- } \\
\text { piece }\end{array}$ & Tail & \\
\hline Guihai, et al. [11] & $\begin{array}{l}\text { Entropy method to extract threshold \& } \\
\text { canny edge detector to obtain the sperm } \\
\text { image }\end{array}$ & $\sqrt{ }$ & $\sqrt{ }$ & $\sqrt{ }$ & $\sqrt{ }$ \\
\hline Berezansky, et al. [8] & Mean-shift algorithm & $\sqrt{ }$ & $\sqrt{ }$ & $\sqrt{ }$ & $\mathrm{X}$ \\
\hline $\begin{array}{l}\text { Shojaedini and Heydari } \\
{[13], \text { Shojaedini and }} \\
\text { Heydari [14] }\end{array}$ & $\begin{array}{l}\text { Combination of entropy of transitions } \\
\text { across boundaries and watershed-based } \\
\text { segmentation }\end{array}$ & $\sqrt{ }$ & $\sqrt{ }$ & $\sqrt{ }$ & $\sqrt{ }$ \\
\hline Vahid Reza, et al. [15] & Best fit ellipses & $\sqrt{ }$ & $\mathrm{X}$ & $\mathrm{X}$ & $\sqrt{ }$ \\
\hline Ravanfar, et al. [12] & $\begin{array}{l}\text { Watershed } \\
\text { thresholding }\end{array}$ & $\sqrt{ }$ & $\mathrm{X}$ & $\mathrm{X}$ & $\sqrt{ }$ \\
\hline Alias, et al. [16] & Template matching & $\sqrt{ }$ & $\mathrm{X}$ & $\mathrm{X}$ & $\mathrm{X}$ \\
\hline Bijar, et al. [9] & $\begin{array}{l}\text { Bayesian classifier with entropy based } \\
\text { expectation maximization and Markov } \\
\text { random field }\end{array}$ & $\sqrt{ }$ & $\sqrt{ }$ & $\sqrt{ }$ & $\sqrt{ }$ \\
\hline Carrillo, et al. [10] & Thresholding \& morphological mask & $\sqrt{ }$ & $\sqrt{ }$ & $\mathrm{X}$ & $\sqrt{ }$ \\
\hline Park, et al. [25] & Strategic Hough transform & $\sqrt{ }$ & $\mathrm{X}$ & $\mathrm{X}$ & $\mathrm{X}$ \\
\hline $\begin{array}{l}\text { Abbiramy and Shanthi } \\
\text { [21] }\end{array}$ & Gradient image \& Watershed algorithm & $\sqrt{ }$ & $\sqrt{ }$ & $\sqrt{ }$ & $\sqrt{ }$ \\
\hline Rahatabad, et al. [19] & Morphological processing & $\sqrt{ }$ & $\mathrm{X}$ & $\mathrm{X}$ & $\sqrt{ }$ \\
\hline Alegre, et al. [18] & Otsu thresholding technique & $\sqrt{ }$ & $\mathrm{X}$ & $\mathrm{X}$ & $\mathrm{X}$ \\
\hline Sánchez, et al. [20] & $\begin{array}{l}\text { Morphological processing \& Otsu } \\
\text { thresholding }\end{array}$ & $\sqrt{ }$ & $\mathrm{X}$ & $\mathrm{X}$ & $\sqrt{ }$ \\
\hline Chang, et al. [7] & $\begin{array}{l}\text { k-means clustering \& histogram } \\
\text { statistical analysis techniques }\end{array}$ & $\sqrt{ }$ & $\mathrm{X}$ & $\mathrm{X}$ & $\sqrt{ }$ \\
\hline Elsayed, et al. [22] & Maximum entropy technique & $\sqrt{ }$ & $\sqrt{ }$ & $\sqrt{ }$ & $\mathrm{X}$ \\
\hline Ghasemian, et al. [24] & $\begin{array}{l}\text { Sobel edge detection \& skeleton } \\
\text { finding }\end{array}$ & $\sqrt{ }$ & $\sqrt{ }$ & $\sqrt{ }$ & $\sqrt{ }$ \\
\hline
\end{tabular}

Recent work by Elsayed, et al. [22] demonstrated an improved computer-assisted sperm analysis plugin for ImageJ to analyze sperm motility in microfluidic environment. The proposed tracking algorithm utilized the existing maximum entropy algorithm [23] and morphological operation to segment the object of interest (i.e. motile sperm) and remove unwanted noises. The tracking algorithm proposed by Elsayed, et al. [22] applied their invention on detecting bull sperms. Although the authors demonstrated the ability of their developed system in producing useful information on motility percentage, their focus is more on the tracking of the motile sperm rather than producing an efficient segmentation tool. Another recent technique published by Ghasemian, et al. [24] presented an effective method in segmenting the human sperm head. Although it is designed for morphology analysis, their published work 
is heavily focuses on the segmenting the sperm (i.e. head, mid-piece and tail) that is more relevant to our study. Denoising algorithm was applied before the sperm region detection process. The sperm was segmented using Sobel edge detection to detect the boundary of the sperm and skeleton finding to detect the backbone of the sperm. This technique has the ability to work in low-resolution images however if the extracted image frame was blurred, semiautomated threshold setting needs to be employed.

In the context of the sperm motility analysis, the segmentation algorithms for sperm analysis were normally presented together with the detection and tracking techniques as summarized in Table 1. Since the importance of the segmentation algorithm will reflect on the sperm detection analysis, an efficient segmentation tool is required to distinguish the sperm from low contrast images as well as determining the discriminative features of the sperms are needed. This is because most of the acquired sperm motility video samples suffer from non-uniform illumination and low contrast regions (could be due by the fast movement of the sperms).

\subsection{METHODS}

The proposed method of an efficient segmentation tool is divided into four main tasks namely variance computation, pixel classification, pixel grouping and threshold computation as illustrated in Fig. 1. The idea of this proposed method is to exclude the unwanted background pixels in the early stage by computing variance of the image. The variance computation is targeted to solve the low contrast issue that normally found in sperm microscopic images during morphology analysis and non-uniform illumination, mostly encountered in video frame of the motility analysis.

Variance is used as the main criteria in this study since it can become an indicator to classify the pixels into ambiguous or unambiguous pixels. This is because pixels with almost similar intensity value will produce low variance (i.e. small intensity value differences). Meanwhile, pixels with high intensity value differences (i.e. intensity variation) will produce high variance. Therefore, by computing variance, the pixels that belong to the background category are directly excluded from subsequent stages and non-uniform illumination issue that could affect the segmentation process is hindered.

The variance image $V_{r}$ is generated by computing the variance of each pixel around its neighbourhood in a predefined local window ( $w \times 1$ w) as shown in Eq. (1) and (2). Then, the normalized variance image is computed by dividing the image by the maximum variance as shown in Eq. (3).

$$
\begin{gathered}
N=w^{2} \\
V_{r}(x, y)=\frac{1}{N-1} \sum_{j=1}^{N}\left(I_{j}-\bar{I}\right)^{2} \\
V_{r_{\text {Normalized }}}(x, y)=\frac{V_{r}(x, y)}{V_{r_{\text {Max }}}}
\end{gathered}
$$

where $N$ is the number of pixels in the local window, $w$ is the window size, $V_{r}(x, y)$ is the variance image, $I_{j}$ is the pixel intensity value, $\bar{I}$ is the average intensity value of the local window, $\operatorname{VIM}_{\text {Normalized }}(x, y)$ is the normalized variance image and $V_{r \text { Max }}$ is the maximum variance from the variance image. Although the intensity distribution of the image is manipulated in the variance computation stage, intensity saturation issue is well handled during normalization process as in Eq. (3). 


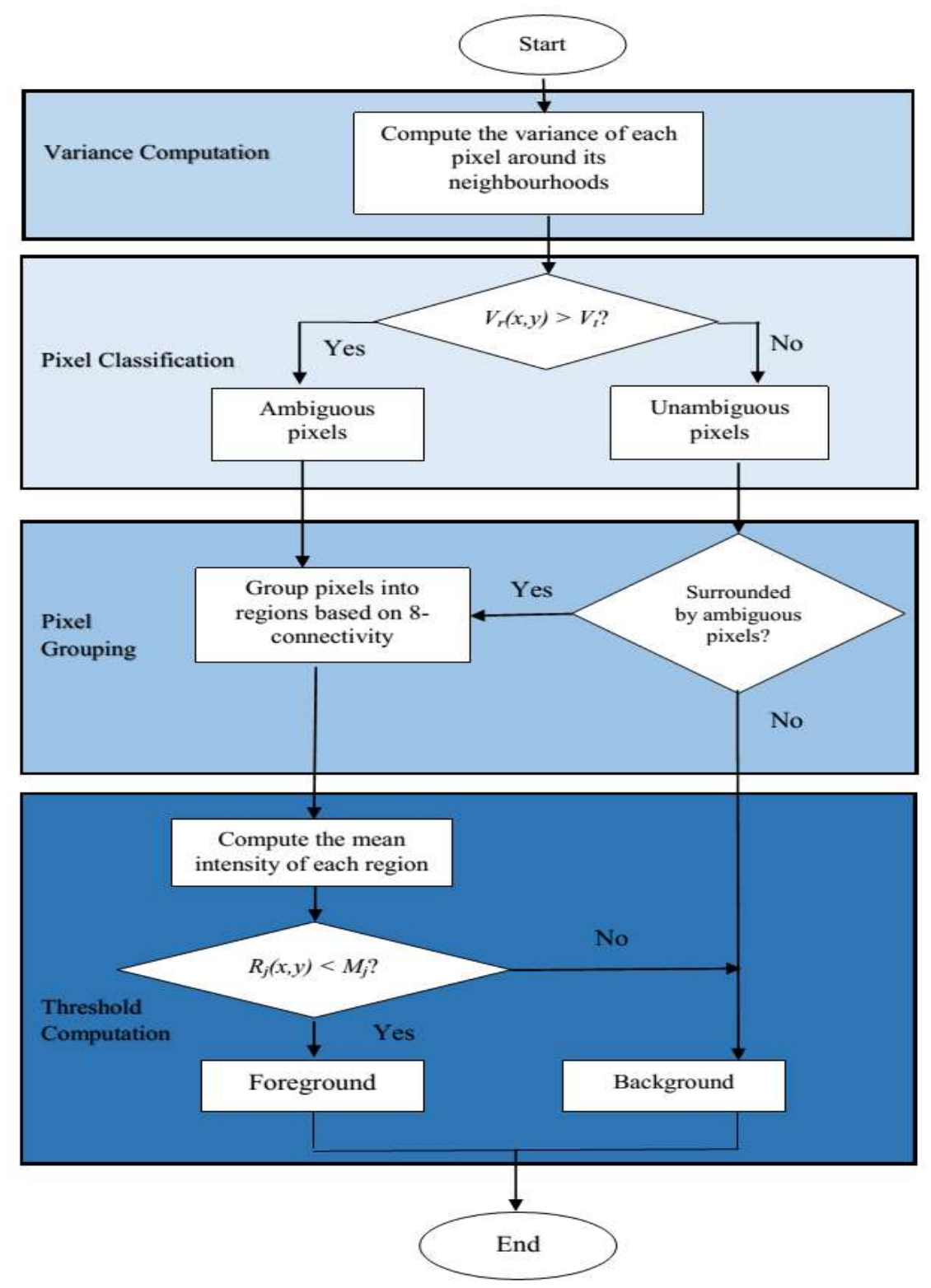

Fig.1: The proposed method of an efficient segmentation tool

Subsequently, all the pixels are classified into two groups based on the threshold intensity variance, $V_{t}$. The $V_{t}$ is defined by the following equations:

$$
\begin{gathered}
V_{t}=\operatorname{SIM}(p, 1) \\
p=\beta\left(N_{T}\right)
\end{gathered}
$$


where $p$ is the position of the threshold intensity variance, $\beta$ is the estimated region occupied by foreground in the image, $N_{T}$ is the total number of image pixel and SIM is the descending sorted array of normalized variance image pixels. The $\beta$ value is ranging from 0 to 1 . It should be more than 0 to avoid the whole image being classified as background and less than 1 to avoid the whole image being classified as foreground. If the chosen $\beta$ is 0.25 , it means that the foreground occupies approximately $25 \%$ of the whole image and the rest of the pixels are assumed as background (this value is chosen in this study since based on the observation, the objects of interest occupy almost $25 \%$ of the whole image)

Fig. 2 shows a sample scenario where white boxes represent background pixels and black boxes represent object pixels. Pixels with intensity value that are higher than the threshold intensity variance are considered as ambiguous pixels and are processed in the next stage. This is shown by the pixels labelled with 'B' in Fig. 2 where they contain both object and background pixels in its $3 \times 3$ local window. Whereas, pixels with intensity value lower than the threshold intensity variance are unambiguous pixels. They can be either object interior pixels or background pixels. The object interior pixel is shown in Fig. 2 denoted by pixel ' $C$ ' and the background pixels are denoted by pixel 'A'. The object interior pixels that are surrounded by ambiguous pixels are filtered out and sent to the next stage with the ambiguous pixels. Eq. (6) summarizes the separation of pixels into two different groups.

$$
V_{r}^{\prime}(x, y)=\left\{\begin{array}{cll}
I_{a m b}(x, y) & \text { if } & V_{r}(x, y)>V_{t} \\
I_{\text {unamb }}(x, y) & \text { if } & V_{r}(x, y) \leq V_{t}
\end{array}\right.
$$

where $V_{r}^{\prime}(x, y)$ is the segmented variance image, $I_{a m b}(x, y)$ is the ambiguous pixel, $I_{\text {unamb }}(x, y)$ is the unambiguous pixel.

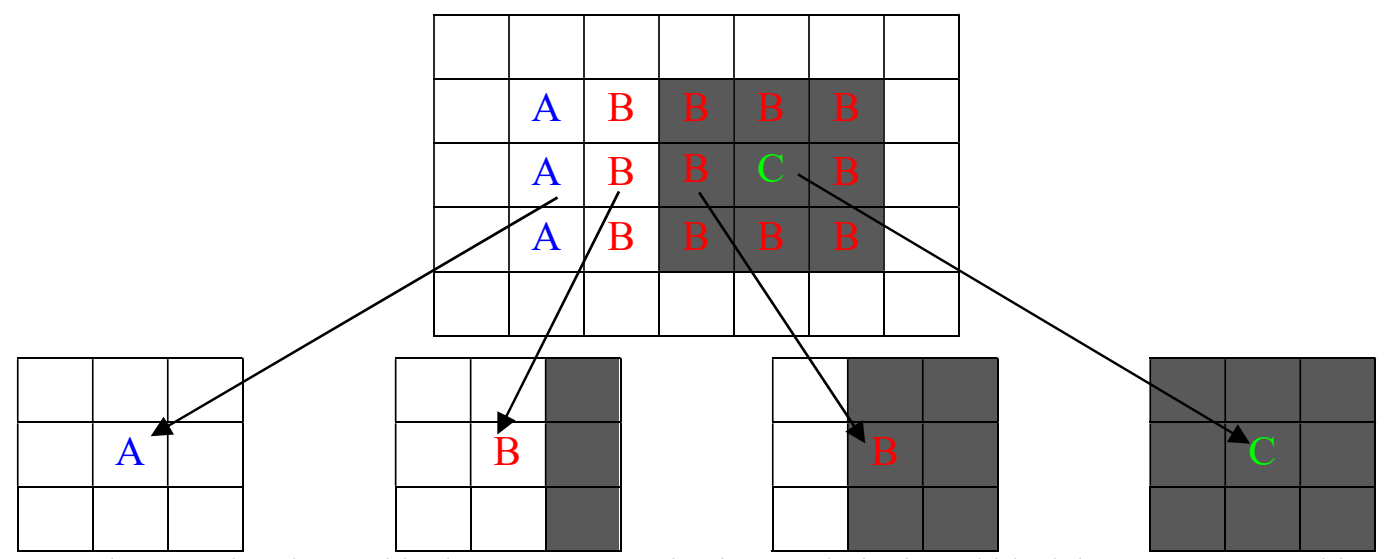

Figure 2 : Sample scenario where white boxes represent background pixels and black boxes represent object pixels

In the next stage, the pixels are grouped into different region, $R_{j}$ based on 8-connectivity. As long as the pixels are 8-connected with each other, they are considered as one region. 8-connected pixels are pixels that touch each other on at least one of their edges or corners. The mean intensity in each region is computed to define the mean threshold for each region as shown in Eq. (7). After that, pixels with intensity lower than the mean threshold are considered as the foreground as described in Eq. (8).

$$
M_{j}=\frac{\sum_{1}^{N} R_{j}(x, y)}{N}
$$




$$
B W(x, y)= \begin{cases}1-\text { Background if } & R_{j}(x, y)>M_{j} \\ 0-\text { Foregrouna if } & R_{j}(x, y) \leq M_{j}\end{cases}
$$

Here, $M_{j}$ is the mean threshold for region $j, N$ is the total number of pixels in region $j$ and $R_{j}(x, y)$ denotes the pixel in region $j . B W(x, y)$ is the binary image that contains only foreground and background pixels.

\subsection{RESULTS}

The performance of the proposed segmentation is presented in this section. The proposed segmentation technique was developed using Matlab R2013a environment in Intel @ ${ }^{\circ}$ Core $^{\mathrm{TM}}$ i7-2600 CPU @3.4GHz. The proposed segmentation technique was tested on Sprague Dawley rat sperm motility video that was collected at Universiti Sains Malaysia Hospital, Kubang Kerian with the video resolution of $389 \times 302$ (grey scale) and frame rate of 30 frames/sec. This paper presents the new segmentation technique which aim to improve the existing segmentation where the whole sperms can be detected. The detection of the whole sperm structure (head, midpiece and tail) is crucial in sperm motility analysis due to the intersected and occluded sperms. It was found in most of the occlusion scenario that the head of the motile sperms are intersected among each other and moved together. The existing motility analysis that normally only focused on the sperm head will neglect the occluded sperms in the analysis and thus inaccurate estimation of sperm velocity could be occurred.

We implemented segmentation technique by Ravanfar, et al. [12] to be included for comparison analysis since it is applied in sperm motility analysis. In addition, we applied proposed technique published by Ghasemian, et al. [24] for comparison since the published work focuses on the segmentation algorithm in detecting morphological of the sperm. Although Ghasemian, et al.'s technique mainly design for morphological analysis, their algorithm able to detect the whole body of the sperm which is relevant to be compared with our method.

The capability of this technique in segmenting the motile sperm in low contrast environment is evaluated and compared with the proposed segmentation technique. The quality of the segmentation is measured by evaluation metrics of non-uniformity (NU). This NU is chosen since it does not require ground-truth image thus reduces the complication of the quantitative analysis. The NU is calculated using [26]:

$$
N U=\frac{\left|F_{T}\right|}{\left|F_{T}+B_{T}\right|} \frac{\sigma_{f}^{2}}{\sigma^{2}}
$$

where $\sigma^{2}$ represents the variance of the whole image, and $\sigma_{f}^{2}$ represents the foreground variance. For a wellsegmented image, it is expected that $N U$ will be closed to ' 0 ' while the worst case of $N U$ will be closed to ' 1 ' corresponds to the segmented image where the background and foreground are indistinguishable.

The proposed method and segmentation technique by Ravanfar, et al. [12] and Ghasemian, et al. [24] were tested on 20 sperm motility video samples where each sample consists of different number of sperms (a total of 327 sperms) as tabulated in Table 2. Fig. 3 shows the segmentation results of the proposed method in the low contrast environment. The left column in this figure shows the original video frames that are low contrast where the intensity of the objects of interest (i.e. motile sperms) are slightly different from their background. For each image, we show the results of applying our proposed method, Ravanfar's method and Ghasemian's method where red arrow indicates the low contrast region. 
Although the visibility of the motile sperms is poor (as pointed by the red arrow), the proposed method is able to segment the whole structure of the motile sperms (i.e. head, mid-piece and tail) as shown in Figs. 3 (b), (f) and (j) respectively. However, the Ravanfar's method is unable to segment the low contrast region as indicated by the red arrow in Figs. 3 (c), (g) and (k) respectively. The results produced by Ghasemian, et al. [24] shows that the unwanted small particles were completely removed from the median filtering process that were applied before the sperms are segmented. However for the occlusion case as shown in Fig. 3 (a), this method tend to dismiss the low contrast region as shown in the red arrow. Although results in Figs. 3(h) and 3(l) illustrated the smoother segmented sperm, the sperm's tail were undetected.

Table 2: Comparison of results for sperm detection between the proposed method, Ravanfar, et al. [12] and Ghasemian, et al. [24]

\begin{tabular}{ccccccccccc}
\hline Sample & \begin{tabular}{c} 
No. of \\
Evaluated \\
\cline { 3 - 9 }
\end{tabular} & \multicolumn{3}{c}{ Ravanfar, et al. [12] } & \multicolumn{3}{c}{ Ghasemian, et al. [24] } & \multicolumn{3}{c}{ Proposed Method } \\
\cline { 3 - 10 } & A (\%) & Sn (\%) & Sp (\%) & A (\%) & Sn (\%) & Sp (\%) & A (\%) & Sn (\%) & Sp (\%) \\
\hline 1 & 13 & 92.12 & 93.65 & 91.24 & 90.13 & 92.14 & 91.64 & 94.03 & 95.45 & 93.67 \\
3 & 24 & 97.85 & 98.12 & 96.32 & 89.32 & 90.87 & 88.32 & 96.83 & 97.52 & 95.28 \\
4 & 23 & 98.15 & 99.62 & 97.98 & 90.62 & 91.62 & 89.61 & 97.98 & 99.24 & 96.15 \\
5 & 12 & 85.23 & 86.34 & 83.96 & 95.32 & 96.32 & 94.28 & 91.23 & 92.54 & 90.87 \\
6 & 25 & 81.24 & 82.54 & 79.61 & 90.32 & 92.14 & 89.69 & 99.58 & 99.89 & 98.95 \\
7 & 20 & 90.63 & 91.24 & 89.87 & 90.56 & 91.30 & 88.62 & 94.23 & 95.21 & 93.01 \\
8 & 14 & 90.23 & 91.68 & 89.74 & 97.32 & 98.65 & 95.27 & 98.93 & 99.12 & 97.69 \\
9 & 23 & 88.21 & 89.21 & 87.36 & 88.25 & 89.27 & 97.24 & 91.23 & 93.04 & 90.54 \\
10 & 13 & 95.41 & 96.04 & 94.87 & 97.32 & 98.64 & 96.34 & 96.21 & 97.67 & 95.34 \\
11 & 10 & 82.36 & 91.62 & 89.87 & 98.24 & 99.38 & 97.65 & 97.64 & 97.99 & 96.32 \\
12 & 12 & 93.1 & 83.51 & 81.2 & 91.23 & 92.04 & 90.51 & 90.45 & 91.65 & 89.87 \\
13 & 9 & 88.67 & 89.65 & 87.65 & 96.42 & 97.02 & 95.64 & 95.65 & 96.67 & 94.98 \\
14 & 12 & 87.64 & 88.62 & 85.34 & 99.32 & 99.56 & 98.53 & 98.79 & 99.12 & 97.98 \\
15 & 23 & 88.32 & 89.65 & 87.61 & 87.65 & 89.02 & 86.57 & 91.56 & 92.45 & 90.78 \\
16 & 14 & 94.23 & 95.38 & 93.79 & 95.26 & 96.57 & 94.32 & 96.87 & 97.67 & 95.89 \\
17 & 15 & 89.67 & 90.21 & 88.67 & 96.87 & 97.65 & 95.32 & 97.62 & 98.65 & 96.24 \\
18 & 13 & 91.24 & 92.14 & 89.24 & 92.32 & 94.65 & 91.54 & 93.45 & 94.67 & 92.78 \\
19 & 28 & 93.16 & 94.06 & 92.89 & 90.31 & 91.20 & 89.87 & 96.15 & 97.67 & 95.87 \\
20 & 18 & 95.23 & 96.85 & 94.97 & 94.32 & 95.62 & 93.75 & 97.67 & 98.67 & 95.75 \\
\hline \hline
\end{tabular}

The segmentation performance of the proposed method in terms of sperm detection is presented in Table 2. For a total of 20 sperm motility video samples, numbers of evaluated sperms are different for each sample and detection accuracy is presented in the table. The sperm detection analysis is considered to appraise the robustness of the proposed method in sperm segmentation. Moreover, the effectiveness of the sperm segmentation will determine the detection of the motile sperm in sperm motility analysis. Overall, the proposed method attains better results than the Ravanfar's and Ghasemian's methods. The Ghasemian's method works best when number of evaluated sperms are small per sample as can be seen in samples 4, 9 and 13. If the number of evaluated sperm is high, tendency of occlusion cases to happen will be increased thus complicates the edge detection process. The Sobel operator in Ghasemian's technique will be affected by the non-uniform illumination in the image and thus complicates the segmentation process. 
In addition to the qualitative observation and comparison of sperm detection results presented in Fig. 3 and Table 2 respectively, the sperm segmentation is also evaluated in terms of NU measurement. NU is the evaluation metrics used to measure the segmentation quality where standard deviations for 20 samples are plotted for each bar as shown in Fig. 4.

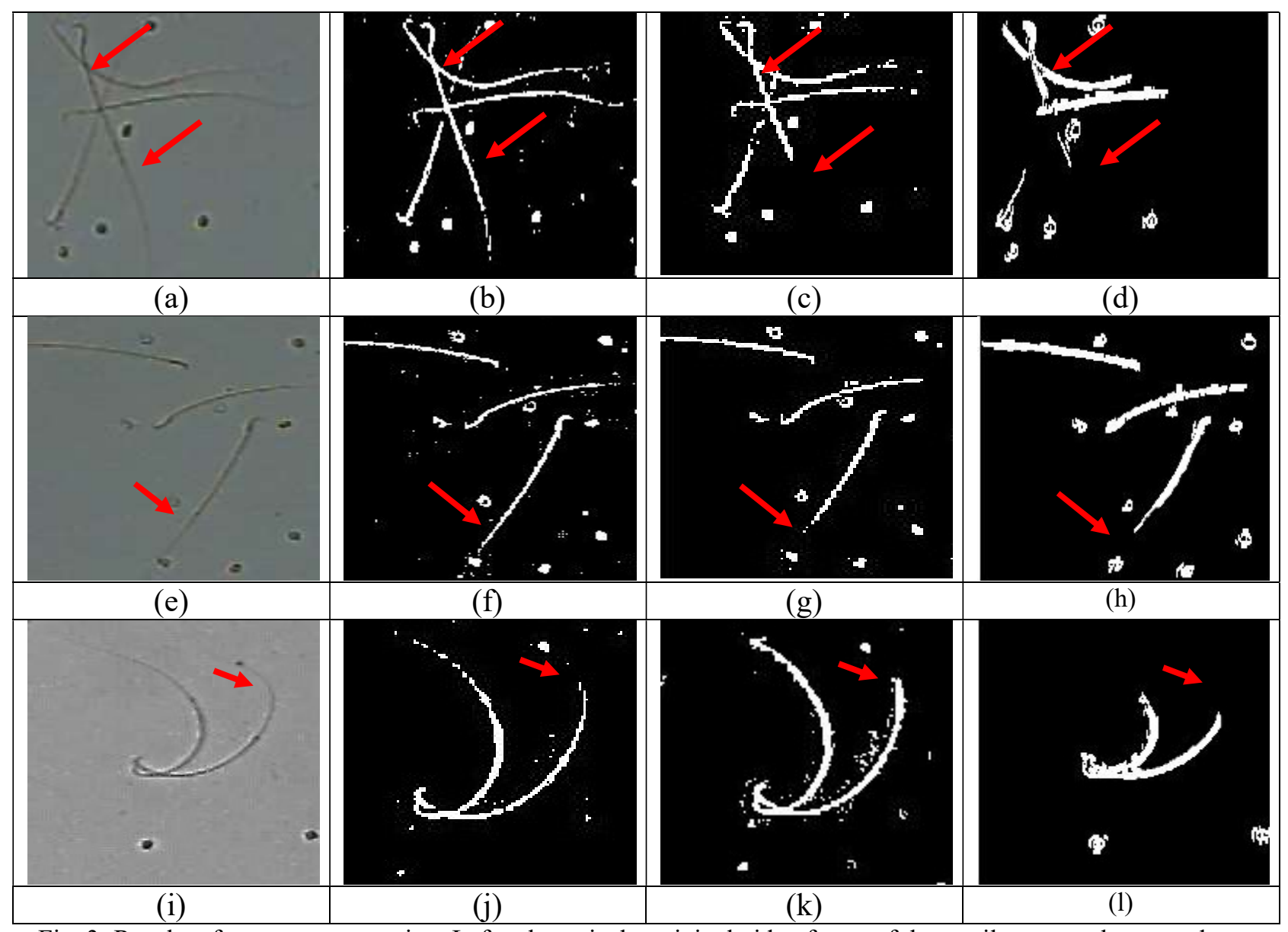

Fig. 3: Results of sperm segmentation. Left column is the original video frame of the motile sperm, the second row is the segmented sperm obtained from the proposed method, third column is the results from Ravanfar, et al. [12] sperm segmentation while the most right column is the results from Ghasemian, et al. [24]

The proposed method attains better results for all sperm samples where the mean and standard deviation of NU is lower than the Ravanfar's method. However for certain samples, the Ghasemian's method attains better result due to the low number of sperms in that sample. These results are in agreement with the qualitative observation in Fig. 3 where the proposed method exhibits better segmentation results even in the low contrast regions. The inability of the Ravanfar's method in detecting the whole structure of the sperm in the low contrast region has affected the sperm detection analysis and thus reducing the accuracy, sensitivity and specificity of the sperm detection analysis. The Ghasemian's method works best with the low number of sperms per sample. This method has shown great potential in segmenting the motile sperm, however due to the low contrast region as well as non-uniform illumination of the image has increased the complexity of segmenting the whole body of the sperms. 


\subsection{DISCUSSIONS}

In this paper we have presented a new Region-based adaptive thresholding technique for motile sperm segmentation attains significant improvement than the current state-of-the-art Ravanfar's and Ghasemian's methods. Our method is different from the other methods in three main aspects:

1. Ability to segment the motile sperms in low contrast environment. In the proposed method, pixels in the image are handled in two stages of pixel classification and pixel-grouping stages as explained in Section 3.0. The pixel classification has grouped the high contrast and low contrast pixels into two groups of ambiguous and unambiguous pixels respectively. The variance computation has enabled the variation of intensity to be computed where low contrast pixels (classified as unambiguous pixels) are grouped and segmented. By computing multiple thresholds in the respective low contrast regions, the whole structure of the sperm can be detected.

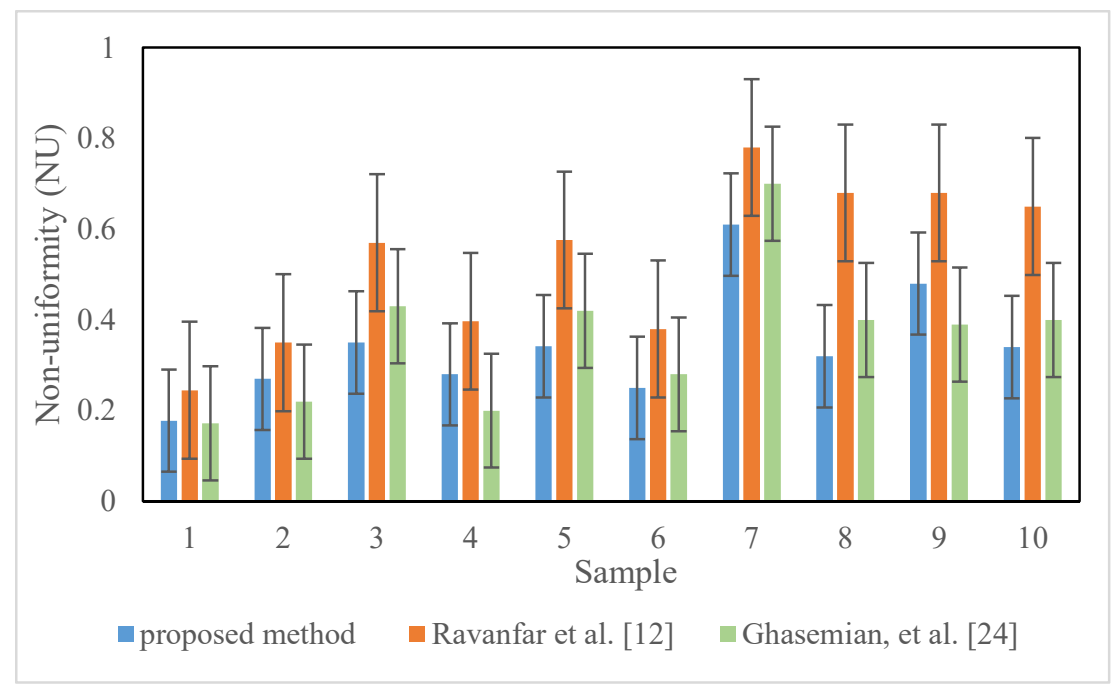

(a)

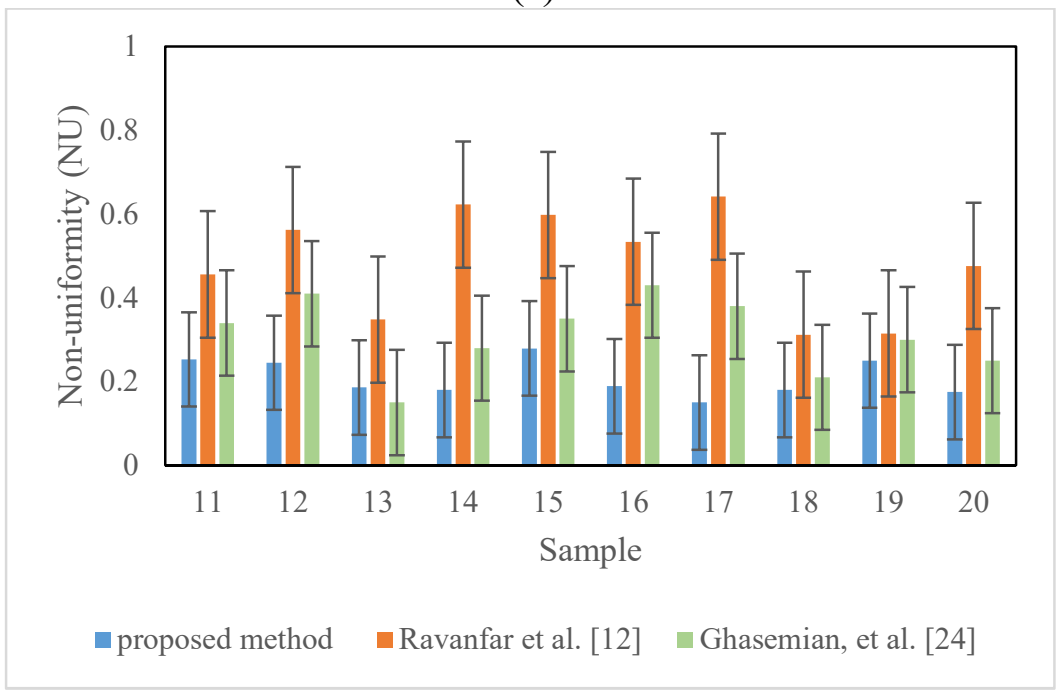

(b)

Figure 4. Results of non-uniformity for 20 samples. The standard deviation of the sample is also plotted for each box. 
Due to the fast movement of the sperm, visibility of the sperm tail is often obscured and neglected in the sperm segmentation. As a result, most available sperm segmentation technique rather focused on sperm head segmentation for their detection and tracking analysis. However, the detection of the whole sperm structure is important especially in occluded scenario as depicted in Figure 3(i). The occlusion of the motile sperms has caused sperm head to be clumped with each other and be considered as one motile sperm. This scenario has affected the sperm detection analysis where the occluded sperms are neglected in the analysis. The Ravanfar's method unable to detect the sperm in low contrast region as depicted by the red arrow in Figure 3(k) as compared to the segmented sperms in Figure 3(j). The ability of the proposed method in segmenting pixels in the low contrast regions has improved the accuracy of the sperm detection that aid the sperm motility tracking. Although the Ghasemian's method produced a smoother segmented sperms as shown in Fig. 3(h), this method had a difficulty in handling the occlusion cases as shown in Figs. 3(d) and 3(1). The Ghasemian's method works better in morphological analysis where an individual sperm is investigated.

2. Does not use pre-processing tool or phase contrast accessories to improve visibility of the sperm. One of the key advantages of the proposed method is its ability to segment the motile sperm without aid of external enhancement tools to improve the visibility of the sperm. Various image enhancement techniques have been implemented prior to the segmentation process (as tabulated in Table 1) since most of the acquired video samples suffered from low contrast and non-uniform illumination. Some of the researchers utilized phase contrast accessories which made the sperm head appear brighter than the other parts. Since the debris in the semen sample and sperm head have similar size, both objects will appear brighter than the other parts which could lead to misidentification of the significant object of interest. The variance computation during the first stage of the proposed segmentation technique has ensured that the deviations of the intensity distribution of the samples are considered. Pixels are grouped and segmented according to their similarity of the intensity distribution where ambiguous and nonambiguous pixels are processed separately. This approach has enabled the low contrast regions to be segmented without the assistance of the pre-processing tools.

3. Use of thresholding method. The existing segmentation approaches are strongly applicant-dependent. Among several segmentation methods, thresholding is a popular choice due to its simplicity and efficiency. Thresholding is a process of binarising an image where lighter pixels are made white and the darker pixels are made black. The low contrast video samples and movement of the sperm have significant impact on the visibility of the motile sperms and thus affecting the sperm segmentation. The multi stages of thresholding approach of variance computation, pixel classification and pixel grouping provide an advantage of segmenting object in low contrast regions without external pre-processing tool. Although the proposed method produced improvement than the Ravanfar's and Ghasemian's methods, this technique is only suitable for motility analysis while specific structure of the sperm head is unable to be identified. An improvement on the existing proposed method to introduce multi-thresholding could aid the identification of the nucleus, acrosome and cytoplasm.

Our results have shown that our approach, based on the aforementioned aspects outperforms the results achieved by other two methods. The results show that the proposed method successfully segments the motile sperm even in the low contrast regions. The qualitative results of the segmented sperms as shown in Figure 3 indicates that more sperm regions are extracted by the proposed method as compared to the Ravanfar's and Ghasemian's methods. We believe it occur mainly because of the results of the pixel classification and pixel grouping stages (as discussed in Section 3) that distinguish the low contrast pixels than the unambiguous pixels. The results obtained from these stages has ensured that the pixel in low contrast regions are segmented separately than the other regions. This process allows a new threshold to be adaptively set according to the ambiguous pixel regions that have almost similar intensity 
distribution. Thus the ambiguous pixels with intensity lower than the new set threshold are considered as the foreground.

It is expected that the accuracy of the sperm detection hardly achieved $100 \%$. Our method is able to attain higher accuracy except for samples 2 and 3 with the average accuracy (out of 20 samples) of $95.74 \%$ as compared to the Ravanfar's method of $90.64 \%$ and Ghasemian's method of $93.42 \%$. There is a trade-off between correct detection for samples 2 and 3 where our method achieves accuracy of $96.83 \%(n=24)$ and $97.98 \%(n=23)$ than the Ravanfar's method that attains $97.85 \%$ and $98.15 \%$ respectively. However for the low number of sperms, the Ghasemian's method achieve better results than our proposed method. This method has the ability to remove the unwanted small particles in the image using median filter prior to the edge detection. The accuracy of the Ghasemian's method for samples 9 and 13 are $97.32 \%$ and $99.32 \%$ respectively as compared to our method that only achieved 96.21 and 98.79 respectively. There is a significant different based on statistical t-test analysis between our method and Ravanfar's method and Ghasemian's method with $p$-value of $0.000145(p<0.05)$ and $0.033829(p<0.05)$ respectively.

The quantitative measurement of NU is also in agreement with the qualitative analysis and sperm detection results. The proposed method attains lower NU that indicates that the foreground can be distinguished from its background. The non-sperm cells and sperms are well segmented including the sperm tail located in the low contrast region. This is because, in the proposed method, image is is adaptively segmented (based on region) and dependency on single threshold for all regions is avoided. Due to the variance computation, small intensity variation in the background can also be detected by our method thus caused the segmentation of the unwanted debris in the resulted image (small segmented objects near sperms). This drawback could easily be solved by applying morphological operation to remove small objects in the image. However, for the comparison of our method with Ravanfar's and Ghasemian's methods that focuses on the performance of the segmentation process, the post-processing step of the morphological operation is not included. Among all the methods, the Ghasemian's method is fast to be computed as compared to our method and Ravanfar's method. The Ghasemian's method only requires an average of $0.25 \mathrm{~s}$ per image frame (with the video resolution of $389 \times 302$ ) while the proposed method requires an average of $0.58 \mathrm{~s}$ per image frame and Ravanfar's method requires an average of $1.21 \mathrm{~s}$ per image frame. The Ravanfar's method requires more computational time since it computes watershed algorithm and thresholding technique to segment the sperms. The comparison of the average processing time was conducted for each image frame and mainly focused on segmentation process. The detection and tracking of the motile sperms requires additional steps that their processing time is not included for comparison.

\subsection{CONCLUSIONS}

We have presented four main stages of a new segmentation approach based on thresholding technique to segment the motile sperm. The generation of the new threshold based on intensity in the classified regions provide an exceptional tool for sperm segmentation in non-uniform and low contrast region. In addition our method has classified the image into regions of ambiguous and non-ambiguous based on variance of intensity distribution of the image. This is very relevant to ensure the sperm tail that are normally low contrast to be segmented and detected which eventually improve the accuracy of the segmentation.

The experimental evaluations based on qualitative observation and quantitative measurement indicate that our proposed method outperforms the state-of-the-art techniques of Ravanfar, et al. [12] and Ghasemian, et al. [24]. Our result achieves notable improvements in the detection accuracy with the average of $95.74 \%$ with NU measurement much lower than the both methods. 
The proposed method has been implemented in the rat sperm samples where only the sperm head are different from the human sperms. Thus the framework of the proposed segmentation method can be easily applied to the human sperm samples. In our future work, this method could has better impact on segmentation if the multi-levels thresholding could be implemented to distinguish the properties of the sperm head for sperm morphology analysis. The complete sperm segmentation tool could be developed which will provide benefit for semen analysis.

\subsection{ACKNOWLEDGEMENT}

This project is supported by the Ministry of Education Malaysia through Fundamental Research Grant Scheme entitled "Formulation of a Robust Framework of Image Enhancement for Non-uniform Illumination and Low Contrast Images" (Reference No: FRGS/1/2014/TK03/USM/02/3) and University of Malaya Research Fund Assistance Grant entitled "Development of a robust framework for medical image segmentation using thresholdingbased segmentation technique" (Reference No: BK057/2014)

\section{REFERENCES}

[1] A. E. Omu, "Sperm Parameters: Paradigmatic Index of Good Health and Longevity," Medical Principles and Practice, vol. 22(suppl 1), 2013.

[2] D. S. Guzick, J. W. Overstreet, P. Factor-Livak, C. K. Brazil, S. t. Nakajima, C. Coutifaris, et al., "Sperm Morphology, Motility and Concentration in Fertile and Infertile Men," The New England Journal of medicine, vol. 345, pp. 1388-1393, 2001.

[3] S. He, K. Jenkins-Keeran, and L. C. Woods, "Activation of Sperm Motility in Striped Bass via a cAMPindependent Pathway," Theriogenology, vol. 61, pp. 1487-1498, 2004.

[4] V. Balakrishnan, R. G. Raj, "Exploring the relationship between urbanized Malaysian youth and their mobile phones: A quantitative approach", Telematics and Informatics, vol. 29, Issue 3, pp. 263-272, 2012, ISSN 0736-5853, http://dx.doi.org/10.1016/j.tele.2011.11.001.

[5] T. Rijsselaere, A. Van Soom, G. Hoflack, D. Maes, and A. de Kruif, "Automated sperm morphometry and morphology analysis of canine semen by the Hamilton-Thorne analyser," Theriogenology, vol. 62, pp. $1292-1306,10 / 1 / 2004$.

[6] M. C. Esteso, E. Rodríguez, A. Toledano-Díaz, C. Castaño, J. Pradiee, A. López-Sebastián, et al., "Descriptive analysis of sperm head morphometry in Iberian ibex (Capra pyrenaica): Optimum sampling procedure and staining methods using Sperm-Class Analyzer®," Animal Reproduction Science, vol. 155, pp. $42-49,4 / / 2015$.

[7] V. Chang, J. M. Saavedra, V. Castañeda, L. Sarabia, N. Hitschfeld, and S. Härtel, "Gold-standard and improved framework for sperm head segmentation," Computer Methods and Programs in Biomedicine, vol. 117, pp. 225-237, 11// 2014.

[8] M. Berezansky, H. Greenspan, D. Cohen-Or, and O. Eitan, "Segmentation and tracking of human sperm cells using spatio- temporal representation and clustering," 2007, pp. 65122M-65122M-12. 
[9] A. Bijar, A. P. Benavent, M. Mikaeili, and R. Khayati, "Fully automatic identification and discrimination of sperm's parts in microscopic images of stained human semen smear " Journal of Biomedical Science and Engineering, vol. 5, pp. 384-395, 2012.

[10] H. Carrillo, J. Villarreal, M. Sotaquira, M. A. Goelkel, and R. Gutierrez, "A Computer Aided Tool for the Assessment of Human Sperm Morphology," in Bioinformatics and Bioengineering, 2007. BIBE 2007. Proceedings of the 7th IEEE International Conference on, 2007, pp. 1152-1157.

[11] L. Guihai, H. Wenming, and L. Song, "2-D maximum entropy spermatozoa image segmentation based on Canny operator," in Intelligent Computing and Integrated Systems (ICISS), 2010 International Conference on, 2010, pp. 243-246.

[12] M. Ravanfar, L. Azinfar, M. H. Moradi, and R. Fazel-Rezai, "Occlusion Robust Low-Contrast Sperm Tracking Using Switchable Weight Particle Filtering," Advances in Sexual Medicine,, vol. 4, pp. 42-54, 2014.

[13] S. V. Shojaedini and M. Heydari, "A New Method for Sperm Characterization for Infertility Treatment: Hypothesis Testing by Using Combination of Watershed Segmentation and Graph Theory," Journal of Medical Signals and Sensors, vol. 4, pp. 274-280, Oct-Dec 02/23/received 09/17/accepted 2014.

[14] S. V. Shojaedini and M. Heydari, "Automatic Sperm Analysis in Microscopic Images of Human Semen: Segmentation Using Minimization of Information Distance," Iranian Journal of Medical Physics, vol. 11, pp. 284-293, 2014.

[15] N. Vahid Reza, M. Mohammad Hasan, and N.-E. Mohammad Hosain, "A template matching algorithm for sperm tracking and classification," Physiological Measurement, vol. 26, p. 639, 2005.

[16] M. Alias, N. Mat Isa, S. Sulaiman, and K. Zamli, "Detection of Sprague Dawley Sperm Using Matching Method," in Knowledge-Based Intelligent Information and Engineering Systems. vol. 5179, I. Lovrek, R. Howlett, and L. Jain, Eds., ed: Springer Berlin Heidelberg, 2008, pp. 541-547.

[17] K. Hasikin and N. A. M. Isa, "Fuzzy enhancement for nonuniform illumination of microscopic Sprague Dawley rat sperm image," in Medical Measurements and Applications Proceedings (MeMeA), 2012 IEEE International Symposium on, 2012, pp. 1-6.

[18] E. Alegre, M. Biehl, N. Petkov, and L. Sánchez, "Automatic classification of the acrosome status of boar spermatozoa using digital image processing and LVQ," Computers in Biology and Medicine, vol. 38, pp. $461-468,4 / / 2008$.

[19] F. N. Rahatabad, M. H. Moradi, and V. R. Nafisi, "A Multi Steps Algorithm for Sperm Segmentation in Microscopic Image " World Academy of Science, Engineering and Technology, vol. 1, pp. 459-461, 2007.

[20] L. Sánchez, N. Petkov, and E. Alegre, "Statistical Approach to Boar Semen Head Classification Based on Intracellular Intensity Distribution," in Computer Analysis of Images and Patterns. vol. 3691, A. Gagalowicz and W. Philips, Eds., ed: Springer Berlin Heidelberg, 2005, pp. 88-95.

[21] V. S. Abbiramy and V. Shanthi, "Spermatozoa Segmentation and Morphological Parameter Analysis Based Detection of Teratozoospermia," International Journal of Computer Applications, vol. 3, pp. 19-23, 2010.

[22] M. Elsayed, T. M. El-Sherry, and M. Abdelgawad, "Development of computer-assisted sperm analysis plugin for analyzing sperm motion in microfluidic environments using Image-J," Theriogenology, vol. 84, pp. 1367-1377, 11// 2015. 
[23] A. Qazi, H. Fayaz, A. Wadi, R. G. Raj, N.A. Rahim, W. A. Khan, "The artificial neural network for solar radiation prediction and designing solar systems: a systematic literature review", Journal of Cleaner Production, vol. 104, pp. 1-12, 2015. ISSN 0959-6526, http://dx.doi.org/10.1016/j.jclepro.2015.04.041.(http://www.sciencedirect.com/science/article/pii/S0959652 615004096).

[24] F. Ghasemian, S. A. Mirroshandel, S. Monji-Azad, M. Azarnia, and Z. Zahiri, "An efficient method for automatic morphological abnormality detection from human sperm images," Computer Methods and Programs in Biomedicine, vol. 122, pp. 409-420, 12// 2015.

[25] K. S. Park, W. J. Yi, and J. S. Paick, "Segmentation of sperms using the strategic Hough transform," Ann Biomed Eng, vol. 25, pp. 294-302, Mar-Apr 1997.

[26] M. Sezgin and B. 1. Sankur, "Survey over image thresholding techniques and quantitative performance evaluation," Journal of Electronic Imaging, vol. 13, pp. 146-168, 2004. 